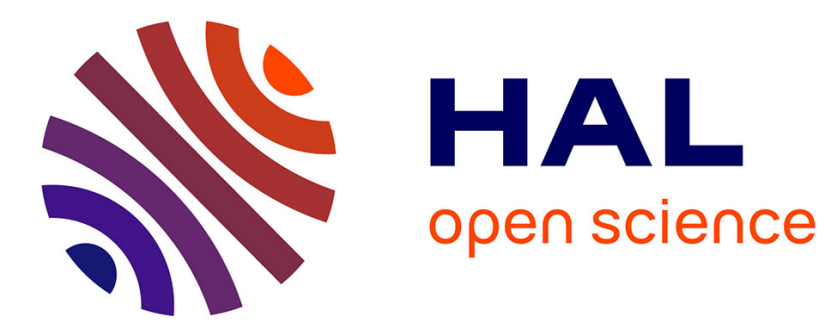

\title{
La Gestion des données naturalistes associatives au service de la gestion des milieux: l'exemple d'Odonat-Alsace.
}

\author{
R. Sane
}

\section{- To cite this version:}

R. Sane. La Gestion des données naturalistes associatives au service de la gestion des milieux: l'exemple d'Odonat-Alsace.. Revue forestière française, 2001, 53 (3-4), pp.404-407. $10.4267 / 2042 / 5256$. hal-03442837

\section{HAL Id: hal-03442837 \\ https://hal.science/hal-03442837}

Submitted on 23 Nov 2021

HAL is a multi-disciplinary open access archive for the deposit and dissemination of scientific research documents, whether they are published or not. The documents may come from teaching and research institutions in France or abroad, or from public or private research centers.
L'archive ouverte pluridisciplinaire HAL, est destinée au dépôt et à la diffusion de documents scientifiques de niveau recherche, publiés ou non, émanant des établissements d'enseignement et de recherche français ou étrangers, des laboratoires publics ou privés. 


\title{
THEmE 3. ECOSYSTÉmES Et BIODIUERSITE FORESTIERE
}

\section{LA GESTION DES DONNÉES NATURALISTES ASSOCIATIVES \\ AU SERVICE DE LA GESTION DES MILIEUX : \\ L'EXEMPLE D’ODONAT-ALSACE}

\author{
Raphä̈l SANÉ
}

\section{HISTORIQUE ET FONCTIONNEMENT}

Créé en 1995, l'Office des DOnnées NATuralistes d'Alsace (ODONAT) est une association à but non lucratif dont l'objet principal est constitué par la connaissance et la protection des espèces animales et végétales et des milieux naturels en Alsace. Cette activité s'exerce au travers de la valorisation des données naturalistes associatives en favorisant la collecte, le traitement et la diffusion des données par les associations membres.

Le fonctionnement d'ODONAT est celui d'une fédération. Seules sont membres des personnes morales dont l'objet est identique à celui d'ODONAT. Des représentants de ces personnes morales composent l'assemblée générale, qui désigne le conseil d'administration puis le bureau; le nombre maximum de représentants par personne morale est le même pour tous les organismes, quelle que soit leur taille.

En pratique, adhérer à ODONAT, c'est souscrire à ses objectifs statutaires et ses principes éthiques. Il n'y a pas transmission obligatoire des informations d'une structure à l'autre, ni centralisation des données. Il y a simplement acceptation de fait de valoriser les données naturalistes. En d'autres termes, les contributions sont toujours libres et volontaires.

ODONAT se situe au centre de la toile naturaliste associative et à l'interface avec les partenaires habituels (État, collectivités, bureaux d'étude, etc.). Il est donc à la fois animateur de réseau et interlocuteur privilégié. Son rôle est essentiellement un rôle de coordination à l'occasion de projets soumis aux associations membres, qu'il s'agisse de projets internes (listes rouges, indicateurs, etc.) ou de sollicitations extérieures (études d’impact, inventaires, etc.).

Les avantages d'un tel réseau sont :

- l'éthique et la déontologie : la propriété des données est un sujet sensible. Les réponses apportées par ODONAT sont simples : respect de la propriété intellectuelle, citation de la source, juste rétribution des producteurs de données... Elles tendent vers une animation démocratique à but non lucratif fondamentalement orienté vers un objectif de protection.

- l'efficience : il est important de pouvoir produire des documents scientifiques de qualité. Pour cela, des outils et moyens informatiques importants sont nécessaires (SIG, bases de données, imprimantes...). L'acquisition et la mise à disposition de tels outils est un des rôles d'ODONAT. 
- l'efficacité : ODONAT a une capacité importante et rapide de réaction face aux demandes. Il doit cela à son intégration dans le réseau naturaliste régional, sans aucune volonté centralisatrice ou de substitution aux associations. Le renforcement de cette efficacité passera par l'harmonisation (en cours) des supports de collecte et de saisie des données.

- l'indépendance : la gestion des données naturalistes constitue aujourd'hui un enjeu essentiel dans plusieurs domaines : gestion du patrimoine naturel, diffusion pertinente de cette connaissance, évaluation objective des politiques de protection. Ces enjeux supposent de pouvoir agir dans un contexte de stricte indépendance par rapport à tout organisme susceptible d'être à la fois juge et partie.

À l'heure actuelle, 26 associations naturalistes sont adhérentes ou correspondantes d'ODONAT sur les 38 environ que compte la région. L'adhésion au principe ODONAT est donc forte. D’autres structures s'inspirant de l'exemple ODONAT voient le jour dans différentes régions françaises (Centre, Rhône-Alpes, Poitou-Charentes...), leur forme s'adaptant au contexte local. Ce développement à l'échelle des régions est coordonné par la fédération France Nature Environnement. Il devrait se concrétiser par la création d'un label de reconnaissance.

\section{ACTIONS}

Les activités d'ODONAT sont de deux ordres :

- favoriser la collecte, le traitement et la diffusion des données naturalistes ;

- assurer le conseil et l'expertise dans le domaine de la nature.

ODONAT existe depuis décembre 1995, mais a connu une montée en puissance en mars 1998 suite à l'embauche d'un salarié. En un peu plus de quatre années d'existence, il a réalisé avec l'aide de son réseau :

- un inventaire des bases de données naturalistes du monde associatif en Alsace (pour le compte de la Région) : ce travail a permis de cerner l'importance du gisement des données naturalistes associatives en Alsace (44 bases différentes dont deux tiers informatisées, 1,3 million de données, 60000 nouvelles données par an, 450 naturalistes) ;

- un état des lieux des ZNIEFF existantes en Alsace (pour le compte de la Région) qui actualise le premier inventaire réalisé dans les années 1980 avant d'engager les ZNIEFF de $2^{\mathrm{e}}$ génération ;

- un inventaire faunistique et floristique de Strasbourg et environs (pour le compte de la Communauté urbaine de Strasbourg) et d'autres travaux de synthèse écologique : animation du réseau naturaliste pour la fourniture de données à des tiers (collectivités, bureaux d'étude) ;

- la mise au point d'un cahier des charges relatif aux conditions de circulation des données : réalisé en consultation avec les principaux partenaires - associatifs ou non -, il vise à clarifier les conditions de diffusion des données associatives (coût, propriété, garanties, spécification...). Cet outil est en phase d'expérimentation ;

- un inventaire des personnes ressources naturalistes : pour pouvoir mieux cerner la nature et l'étendue des ressources naturalistes en France, l'Institut français de l'Environnement (IFEN) a chargé le Muséum national d'Histoire naturelle (MNHN) de mettre au point un protocole d'enquête. ODONAT a été désigné pour tester ce protocole d'inventaire au niveau des associations alsaciennes. Cette enquête a permis de recenser plus de 450 naturalistes avec leurs spécialités, leur aire d'action, leur fréquence de sortie, etc. 
- la réalisation de la bibliographie commentée de l'avifaune alsacienne, par Yves Muller, sur le même modèle de celles existantes en Lorraine et en France.

D'autres actions sont en voie d'achèvement :

- La réalisation de listes rouges des espèces et des milieux en Alsace : les listes rouges sont des outils essentiels dans l'aide à la conservation des espèces et des milieux. Elles s'adressent à un public large (politiques, gestionnaires, aménageurs, bureaux d'étude...). Elles concerneront une dizaine de groupes taxonomiques et seront rédigées par les associations compétentes. Publication prévue début 2002 .

- La publication de la thèse du Professeur Roland Carbiener sur la végétation des Hautes Vosges ainsi que d'un ouvrage de vulgarisation à partir de ce mémoire.

Enfin, les principaux projets d'ODONAT sont les suivants :

- Structuration du réseau : ODONAT souhaite mettre en œuvre un système conceptuel et logistique cohérent de gestion des données à destination de ses associations membres. Ce cadre méthodologique commun de collecte et de formatage des données sera utilisable en réseau et compatible dans son principe avec toutes les bases de données existantes et semblables.

- Élaboration d'indicateurs écologiques de qualité des milieux : l'évaluation de la pertinence des mesures de conservation des milieux est essentielle pour qui souhaite s'interroger sur le bienfondé des actions menées dans ce domaine. Elle passe par l'élaboration d'indicateurs écologiques fiables de la qualité des milieux. ODONAT projette d'élaborer de tels indicateurs sur la base des compétences et connaissances multidisciplinaires qu'elle fédère.

- Inventaire ZNIEFF $2^{\mathrm{e}}$ génération, suivi des sites Natura 2000 , etc. : l'étendue, la densité et les compétences du réseau ODONAT le désignent naturellement pour effectuer l'inventaire ou le suivi écologique de sites naturels patrimoniaux.

Enfin, ODONAT est reconnu comme expert dans le domaine de la nature et participe à diverses instances consultatives (comités de gestion, groupes de travail...).

\section{UTILISATION DES DONNÉES DE L'IFN PAR LES ASSOCIATIONS NATURALISTES}

Dans le domaine de la gestion forestière, les actions d'ODONAT existent sous forme de projets à court et moyen terme.

Le premier est la réalisation de listes rouges des animaux, végétaux et habitats menacés d'Alsace, dont une bonne partie est liée au milieu forestier. L'utilité de ce type d'outil en matière d'aide à la décision n'est plus à démontrer.

Le second est l'élaboration d'indicateurs de qualité des milieux, permettant un suivi et une caractérisation des écosystèmes ainsi qu'une évaluation de leur fonctionnement. Leur mise au point est notamment prévue dans le cadre des nouvelles Orientations régionales forestières et de la certification des forêts.

Concrètement, l'utilisation des données de l'IFN est rendue problématique dans le cadre de ces projets par au moins deux inconvénients majeurs :

- le caractère trop ponctuel des relevés, aussi bien dans l'espace (placettes de $25 \mathrm{~m}$ ) que dans le temps (un passage par placette par campagne). Ce protocole est inadapté pour donner une image suffisamment exhaustive des caractéristiques écologiques et floristiques des milieux, 
exploitable par les associations naturalistes ; en particulier, il ne permet pas de relever les hétérogénéités dans les peuplements, ni les éléments de faible occurrence ;

- le taux d'actualisation des relevés (campagnes décennales) est beaucoup trop faible pour les besoins naturalistes associatifs; en effet, ces derniers effectuent une collecte en continu des données de terrain, alimentant les bases en flux tendu. Cela permet une mise à jour régulière des travaux de synthèses, ainsi qu'une réponse immédiate en cas de besoins d'informations actuelles. 